\title{
Detecting Human-Object Interactions via Functional Generalization
}

\author{
Ankan Bansal, Sai Saketh Rambhatla, Abhinav Shrivastava, Rama Chellappa \\ University of Maryland, College Park \\ \{ankan, rssaketh, abhinav, rama\}@umiacs.umd.edu
}

\begin{abstract}
We present an approach for detecting human-object interactions (HOIs) in images, based on the idea that humans interact with functionally similar objects in a similar manner. The proposed model is simple and efficiently uses the data, visual features of the human, relative spatial orientation of the human and the object, and the knowledge that functionally similar objects take part in similar interactions with humans. We provide extensive experimental validation for our approach and demonstrate state-of-the-art results for HOI detection. On the HICO-Det dataset our method achieves a gain of over $2.5 \%$ absolute points in mean average precision (mAP) over stateof-the-art. We also show that our approach leads to significant performance gains for zero-shot HOI detection in the seen object setting. We further demonstrate that using a generic object detector, our model can generalize to interactions involving previously unseen objects.
\end{abstract}

\section{Introduction}

Human-object interaction (HOI) detection is the task of localizing and inferring relationships between a human and an object, e.g., "eating an apple" or "riding a bike." Given an input image, the standard representation for HOIs (Sadeghi and Farhadi 2011; Gupta and Malik 2015) is a triplet 〈human, predicate, object), where human and object are represented by bounding boxes, and predicate is the interaction between this (human, object) pair. At first glance, it seems that this problem is a composition of the atomic problems of human and object detection and HOI classification (Shen et al. 2018; Gkioxari et al. 2017). These atomic recognition tasks are certainly the building blocks of a variety of approaches for HOI understanding (Shen et al. 2018; Delaitre, Sivic, and Laptev 2011); and the progress in these atomic tasks directly translates to improvements in HOI understanding. However, the task of HOI understanding comes with its own unique set of challenges (Lu et al. 2016; Chao et al. 2017).

These challenges are due to the combinatorial explosion of the possible interactions with increasing number of objects and predicates. For example, in the commonly used

Copyright (c) 2020, Association for the Advancement of Artificial Intelligence (www.aaai.org). All rights reserved.

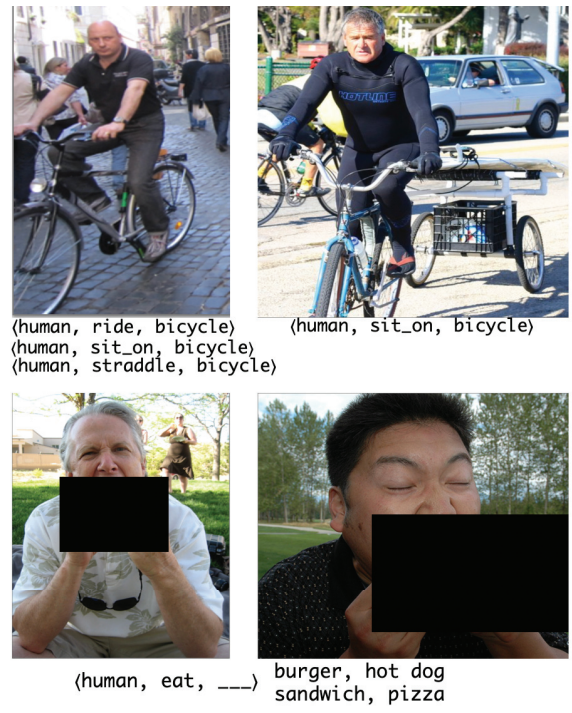

Figure 1: Common properties of HOI Detection. Top Datasets are not exhaustively labeled. Bottom - Humans interact similarly with functionally similar objects - both persons could be eating either a burger, a hot dog, or a pizza.

HICO-Det dataset (Chao et al. 2017) with 80 unique object classes and 117 predicates, there are 9,360 possible relationships. This number increases to more than $10^{6}$ for larger datasets like Visual Genome (Krishna et al. 2017) and HCVRD (Zhuang et al. 2017b), which have hundreds of object categories and thousands of predicates. This, combined with the long-tail distribution of HOI categories, makes it difficult to collect labeled training data for all HOI triplets. A common solution to this problem is to arbitrarily limit the set of HOI relationships and only collect labeled images for this limited subset. For example, the HICO-Det benchmark has only 600 unique relationships.

Though these datasets can be used for training models for recognizing a limited set of HOI triplets, they do not address the problem completely. For example, consider the images shown in Figure 1 (top row) from the challenging HICO-Det dataset. The three pseudo-synonymous rela- 
tionships: 〈human, hold, bicycle〉, 〈human, sit_on, bicycle), and 〈human, straddle, bicycle) are all possible for both these images; but only a subset is labeled for each. We argue that this is not a quality control issue while collecting a dataset, but a problem associated with the huge space of possible HOI relationships. It is enormously challenging to exhaustively label even the 600 unique HOIs, let alone all possible interactions between humans and objects. An HOI detection model that relies entirely on labeled data will be unable to recognize the relationship triplets that are not present in the dataset, but are common in the realworld. For example, a naïve model trained on HICO-Det cannot recognize the 〈human, push, car $\rangle$ triplet because this triplet does not exist in the training set. The ability to recognize previously unseen relationships (zero-shot recognition) is a highly desirable capability for HOI detection.

In this work, we address the challenges discussed above using a model that leverages the common-sense knowledge that humans have similar interactions with objects that are functionally similar. The proposed model can inherently do zero-shot detection. Consider the images in Figure 1 (second row) with (human, eat, ?〉 triplet. The person in either image could be eating a burger, a sandwich, a hot dog, or a pizza. Inspired by this, our key contribution is incorporating this common-sense knowledge in a model for generalizing HOI detection to functionally similar objects. This model utilizes visual appearance of a human, their relative geometry with the object, and language priors (Mikolov et al. 2013) to capture which objects afford similar predicates (Gibson 1979). Such a model is able to exploit the large amount of contextual information present in the language priors to generalize HOIs across functionally similar objects.

In order to train this module, we need a list of functionally similar objects and labeled examples for the relevant HOI triplets, neither of which are readily available. To overcome this, we propose a way to train this model by: 1) using a large vocabulary of objects, 2) discovering functionally similar objects automatically, and 3) proposing dataaugmentation, emulating the examples shown in Figure 1 (second row). To discover functionally similar objects in an unsupervised way, we use a combination of visual appearance features and semantic word embeddings (Mikolov et al. 2013) to represent the objects in a "world set" (Open Images Dataset (OID) (Kuznetsova et al. 2018)). Note that the proposed method is not contingent on the world set. Any large dataset, like ImageNet, could replace OID. Finally, to emulate the examples shown in Figure 1 (second row), we use the human and object bounding boxes from a labeled interaction, the visual features from the human bounding box, and semantic word embeddings of all functionally similar objects. Notice that this step does not utilize the visual features for objects, just their relative locations with respect to a human, enabling us to perform this data-augmentation. Further, to efficiently use the training data, we fine-tune the object detector on the HICO-Det dataset unlike prior approaches.

The proposed approach achieves over $2.5 \%$ absolute improvement in mAP over the best published method for HICO-Det. Further, using a generic object detector, and the proposed functional generalization model lends itself di- rectly to the zero-shot HOI triplet detection problem. We clarify that zero-shot detection is the problem of detecting HOI triplets for which the model has never seen any images. Knowledge about functionally similar objects enables our system to detect interactions involving objects not contained in the original training set. Using just this generic object detector, our model achieves state-of-the-art performance for HOI detection on the popular HICO-Det dataset in the zero-shot setting, improving over existing methods by several percentage points. Additionally, we show that the proposed approach can be used as a way to deal with social/systematic biases present in vision+language datasets (Zhao et al. 2017; Anne Hendricks et al. 2018).

In summary, the contributions of this paper are: (1) a functional generalization model for capturing functional similarities between objects; (2) a method for training the proposed model; and (3) state-of-the-art results on HICO-Det in both fully-supervised and zero-shot settings.

\section{Related Work}

Human-Object Interaction. Early methods (Yao and FeiFei 2010; Yao et al. 2011) relied on structured visual features which capture contextual relationships between humans and objects. Similarly, (Delaitre, Sivic, and Laptev 2011) used structured representations and spatial co-occurrences of body parts and objects to train models for HOI recognition. Gupta et al. (Gupta and Davis 2007; Gupta, Kembhavi, and Davis 2009) adopted a Bayesian approach that integrated object classification and localization, action understanding, and perception of object reaction. (Desai and Ramanan 2012) constructed a compositional model which combined skeleton models, poselets, and visual phrases.

More recently, with the release of large datasets like HICO (Chao et al. 2015), Visual Genome (Krishna et al. 2017), HCVRD (Zhuang et al. 2017b), V-COCO (Gupta and Malik 2015), and HICO-Det (Chao et al. 2017), the problem of detecting and recognizing HOIs has attracted significant attention. This has been driven by HICO which is a benchmark dataset for recognizing human-object interactions. The HICO-Det dataset extended HICO by adding bounding box annotations. V-COCO is a much smaller dataset containing 26 classes and about 10,000 images. On the other hand, HCVRD and Visual Genome provide annotations for thousands of relationship categories and hundreds of objects. However, they suffer from noisy labels. We primarily use the HICO-Det dataset to evaluate our approach in this paper.

(Gkioxari et al. 2017) designed a system which trains object and relationship detectors simultaneously on the same dataset and classifies a human-object pair into a fixed set of pre-defined relationship classes. This precludes the method from being useful for detecting novel relationships. (Xu et al. 2018) used pose and gaze information for HOI detection. (Kolesnikov, Lampert, and Ferrari 2018) introduced the Box Attention module to a standard R-CNN and trained simultaneously for object detection and relationship triplet prediction. Graph Parsing Neural Networks (Qi et al. 2018) incorporated structural knowledge and inferred a parse graph in a message passing inference framework. In contrast, our 
method does not need iterative processing and requires only a single pass through a neural network.

Unlike most prior work, we do not directly classify into a fixed set of relationship triplets but into predicates. This helps us detect previously unseen interactions. The method closest in spirit to our approach is (Shen et al. 2018) which uses a two branch structure with the first branch responsible for detecting humans and predicates, and the second for detecting objects. Unlike our proposed approach, their method solely depends on the appearance of the human. Also, they do not use any prior information from language. Our model utilizes implicit human appearance, the object label, humanobject geometric relationship, and knowledge about similarities between objects. Hence, our model achieves much better performance than (Shen et al. 2018).

We also distinguish our work from prior work (Kato, Li, and Gupta 2018; Fang et al. 2018) on HOI recognition. We tackle the more difficult problem of detecting HOIs here.

Zero-shot Learning. Our work also ties well with zeroshot classification (Xian, Schiele, and Akata 2017; Kodirov, Xiang, and Gong 2017) and zero-shot object detection (ZSD) (Bansal et al. 2018). (Bansal et al. 2018) proposed projecting images into the word-vector space to exploit the semantic properties of such spaces. They also discussed challenges associated with training and evaluating ZSD. A similar idea was used in (Kodirov, Xiang, and Gong 2017) for zero-shot classification. (Rahman, Khan, and Porikli 2018), on the other hand, used meta-classes to cluster semantically similar classes. In this work, we also use wordvectors as semantic information for our generalization module. This, along with our approach for generalization during training, helps zero-shot HOI detection.

\section{Approach}

Figure 2 represents our approach. The main novelty of our proposed approach lies in incorporating generalization through a language component. This is done by using functional similarities of objects during training. For inference, we first detect humans and objects in the image using our object detectors, which also give the corresponding (RoI-pooled (Ren et al. 2015)) feature representations. Each human-object pair is used to extract visual and language features which are used to predict the predicate associated with the interaction. We describe each component of the model and the training procedure in the following sections.

\section{Object Detection}

In the fully-supervised setting, we use an object detector fine-tuned on the HICO-Det dataset. For zero-shot detection and further experiments, we use a Faster-RCNN (Huang et al. 2017) based detector trained on the Open Images dataset (OID) (Kuznetsova et al. 2018). This network can detect 545 object categories and we use it to obtain proposals for humans and objects in an image. The object detectors also output the ROI-pooled features corresponding to these detections. All human-object pairs thus obtained are passed to our model which outputs probabilities for each predicate.

\section{Functional Generalization Module}

Humans look similar when they interact with functionally similar objects. Leveraging this fact, the functional generalization module exploits object similarities, the relative spatial location of human and object boxes, and the implicit human appearance to estimate the predicate. At its core, it comprises a Multi Layer Perceptron (MLP), which takes as input the human and object word embeddings, $w_{h}$ and $w_{o}$, the geometric relationship between the human and object boxes $f_{g}$, and the human visual feature $f_{h}$. The geometric feature is useful as the relative positions of a human and an object can help eliminate certain predicates. The human feature $f_{h}$ is used as a representation for the appearance of the human. This appearance representation is added because the aim is to incorporate the idea that humans look similar while interacting with similar objects. For example, a person drinking from a cup looks similar while drinking from a glass or a bottle. The four features $w_{h}, w_{o}, f_{g}$, and $f_{h}$ are concatenated and passed through a 2-layer MLP which predicts the probabilities for each predicate. All the predicates are considered independent. We now give details of different components in this model.

Word embeddings. We use 300-D vectors from word 2 vec (Mikolov et al. 2013) to get the human and object embeddings $w_{h}$ and $w_{o}$. Object embeddings allow discovery of previously unseen interactions by exploiting semantic similarities between objects. The human embedding, $w_{h}$, helps in distinguishing between different words for humans (man/woman/boy/girl/person), if required.

Geometric features. Following prior work on visual relationship detection (Zhuang et al. 2017a), we define the geometric relationship feature as:

$$
\begin{array}{r}
f_{g}=\left[\frac{x_{1}^{h}}{W}, \frac{y_{1}^{h}}{H}, \frac{x_{2}^{h}}{W}, \frac{y_{2}^{h}}{H}, \frac{A^{h}}{A^{I}}, \frac{x_{1}^{o}}{W}, \frac{y_{1}^{o}}{H}, \frac{x_{2}^{o}}{W}, \frac{y_{2}^{o}}{H}, \frac{A^{o}}{A^{I}},\right. \\
\left(\frac{x_{1}^{h}-x_{1}^{o}}{x_{2}^{o}-x_{1}^{o}}\right),\left(\frac{y_{1}^{h}-y_{1}^{o}}{y_{2}^{o}-y_{1}^{o}}\right), \\
\left.\quad \log \left(\frac{x_{2}^{h}-x_{1}^{h}}{x_{2}^{o}-x_{1}^{o}}\right), \log \left(\frac{y_{2}^{h}-y_{1}^{h}}{y_{2}^{o}-y_{1}^{o}}\right)\right]
\end{array}
$$

where, $W, H$ are the image width and height, $\left(x_{i}^{h}, y_{i}^{h}\right)$, and $\left(x_{i}^{o}, y_{i}^{o}\right)$ are the human and object bounding box coordinates respectively, $A^{h}$ is the area of the human box, $A^{o}$ is the area of the object box, and $A^{I}$ is the area of the image. The geometric feature $f_{g}$ uses spatial features for both entities (human and object) and also spatial features from their relationship. It encodes the relative positions of the two entities.

Generalizing to new HOIs. We incorporate the idea that humans interacting with similar objects look similar via the functional generalization module. As shown in figure 3, this idea can be added by changing the object name while keeping the human word vector $w_{h}$, the human visual feature $f_{h}$, and the geometric feature $f_{g}$ fixed. Each object has a different word-vector and the model learns to recognize the same predicate for different human-object pairs. Note that this does not need visual examples for all human-object pairs. 


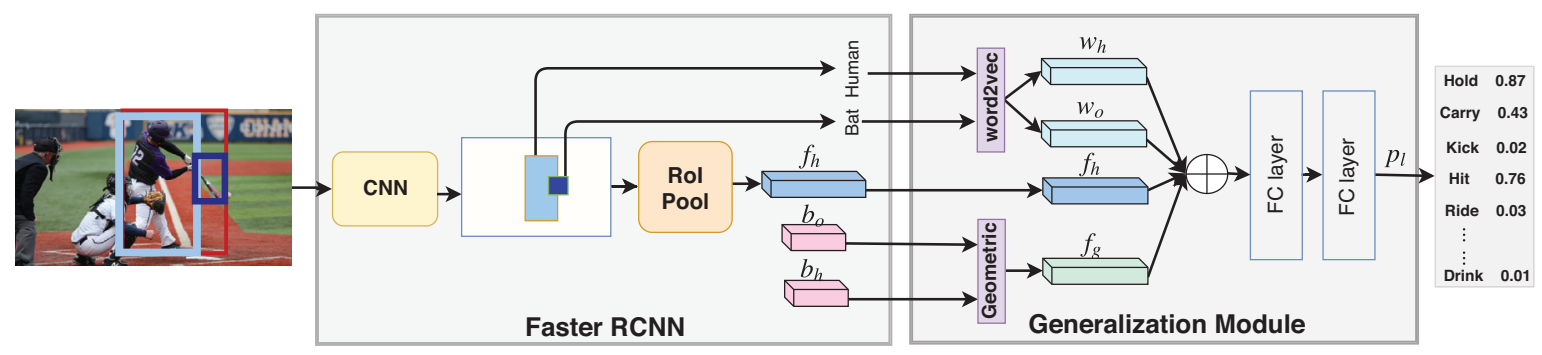

Figure 2: We detect all objects and humans in an image. This detector gives human features $f_{h}$, and the corresponding labels. We consider all pairs of human-object and create union boxes. Our functional generalization module uses the word vectors for the human $w_{h}$, the object class $w_{o}$, geometric features $f_{g}$, and $f_{h}$ to produce the probability estimate over the predicates.

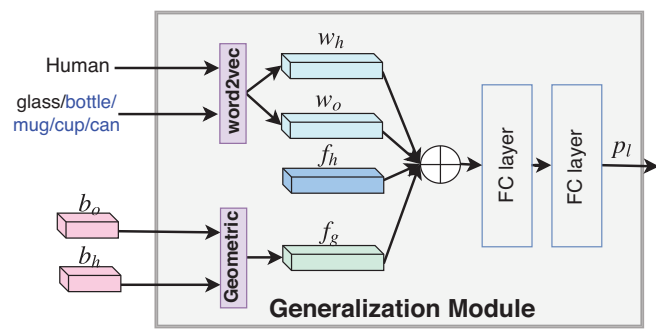

Figure 3: Generalization module. During training, we can replace "glass" by "bottle", "mug", "cup", or "can".

Finding similar objects. A naïve choice for defining similarity between objects would be through the WordNet hierarchy (Miller 1995). However, several issues make using WordNet impractical. The first is defining distance between the nodes in the tree. The height of a node cannot be used as a metric because different things have different levels of categorization in the tree. Similarly, defining sibling relationships which adhere to functional intuitions is challenging. Another issue is the lack of correspondence between closeness in the tree and semantic similarities between objects.

To overcome these problems, we consider similarity in both the visual and semantic representations of objects. We start by defining a vocabulary of objects $\mathcal{V}=\left\{o_{1}, \ldots, o_{n}\right\}$ which includes all the objects that can be detected by our object detector. For each object $o_{i} \in \mathcal{V}$, we obtain a visual feature $f_{o_{i}} \in \mathbb{R}^{p}$ from images in OID, and a word vector $w_{o_{i}} \in \mathbb{R}^{q}$. We concatenate these two to obtain the mixed representation $u_{o_{i}}$ for object $o_{i}$. We then cluster $u_{i}$ 's into $K$ clusters using Euclidean distance. Objects in the same cluster are considered functionally similar. This clustering has to be done only once. We use these clusters to find all objects similar to an object in the target dataset. Note that there might not be any visual examples for many of the objects obtained using this method. This is why we do not use the RoI-pooled visual features from the object.

Using either just the word2vec representations or just the visual representations for clustering gave several inconsistent clusters. Therefore, we use the concatenated features $u_{o_{i}}$. We observed that clusters created using these features better correspond to functional similarities between objects. Generating training data. For each relationship triplet $<h, p, o\rangle$ in the original dataset, we add $r$ triplets $\left\langle h, p, o_{1}\right\rangle$, $<h, p, o_{2}>, \ldots,<h, p, o_{r}>$ to the dataset keeping the human, and object boxes fixed, and only changing the object name. This means that, for all these $f_{g}$ and $f_{h}$ are the same as for the original sample. The $r$ different objects, $o_{1}, \ldots, o_{r}$ belong to the same cluster as object $o$. For example, in figure 3 , the ground truth category "glass" can be replaced by "bottle", "mug", "cup", or "can" while keeping $w_{h}, f_{h}$, and $f_{g}$ fixed.

\section{Training}

A training batch consists of $T$ interaction triplets. The model produces probabilities for each predicate independently. We use a weighted class-wise BCE loss for training the model.

Noisy labeling. Missing and incorrect labels are a common issue in HOI datasets. Also, a human-object pair can have different types of interactions at the same time. For example, a person can be sitting on a bicycle, riding a bicycle, and straddling a bicycle. These interactions are usually labeled with slightly different bounding boxes. To overcome these issues, we use a per-triplet loss weighing strategy. A training triplet in our dataset has a single label, e.g. <human-ride-bicycle>. A triplet with slightly shifted bounding boxes might have another label, like $\langle$ human-sit_on-bicycle $>$. The idea is that the models should be penalized more if they fail to predict the correct class for a triplet. Given the training sample <human-ride-bicycle>, we want the model to definitely predict "ride", but we should not penalize it for predicting "sit_on" as well. Therefore, while training the model, we use the following weighing scheme for classes. Suppose that a training triplet is labeled <human-ride-bicycle $>$ and there are some other triplets in the image. For this training triplet, we assign a high weight (10.0 here) to the loss for the correct class ( $r i d e$ ), and a zero weight to all other predicates in the image. We also scale down the weight (1.0 here) to the loss for all other classes to ensure that the model is not penalized too much for predicting a missing but correct label.

\section{Inference}

The inference step is simply a forward pass through the network (figure 2). The final step of inference is class-wise nonmaximal suppression (NMS) over the union of human and 
object boxes. This helps in removing multiple detections for the same interaction and leads to higher precisions.

\section{Experiments}

We evaluate our approach on the HICO-Det dataset (Chao et al. 2015). As mentioned before, V-COCO (Gupta and Malik 2015) is a small dataset and does not provide any insights into the proposed method. In line with recent work (Gupta, Schwing, and Hoiem 2019), we avoid using it.

\section{Dataset and Evaluation Metrics}

HICO-Det extends the HICO dataset (Chao et al. 2015) which contains $600 \mathrm{HOI}$ categories for 80 objects. HICODet adds bounding box annotations for humans, and objects for each HOI category. The training set contains over 38,000 images and about 120,000 HOI annotations for the $600 \mathrm{HOI}$ classes. The test set has 33,400 HOI instances.

We use mean average precision (mAP) commonly used in object detection. An HOI detection is considered a true positive if the minimum of human overlap $\mathrm{IOU}_{h}$ and object overlap $\mathrm{IOU}_{o}$ with the ground truths is greater than 0.5. Performance is usually reported for three different HOI category sets: (a) all 600 classes (Full), (b) 138 classes with less than 10 training samples (Rare), and (c) the remaining 462 classes with more than 10 training samples (Non-Rare).

\section{Implementation Details}

We start with a ResNet-101 backbone Faster-RCNN which is fine-tuned for the HICO-Det dataset. This detector was originally trained on COCO (Lin et al. 2014) which has the same 80 object categories as HICO-Det. We consider all detections for which the detection confidence is greater than 0.9 and create human-object pairs for each image. Each detection has an associated feature vector. These pairs are then passed through our model. The human feature $f_{h}$ is 2048 dimensional. The two hidden layers in the model are of dimensions 1024 and 512. The model outputs probability estimates for each predicate independently and the final output prediction is all predicates with probability $\geq 0.5$. We report performance with the $\mathrm{COCO}$ detector in supplementary.

For all the experiments, we train the model for 25 epochs with 0.1 initial learning rate which is dropped by a tenth every 10 epochs. We re-iterate that the object detector and the word2vec vectors are frozen while training this model. For all experiments we use up to five $(r)$ additional objects for augmentation, i.e., for each human-object pair in the training set, we add up to five objects from the same cluster while leaving the bounding boxes and human features unchanged.

\section{Results}

With no functional generalization, our baseline model achieves an mAP of $12.17 \%$ for Rare classes which is already higher than all but the most recent methods. This is because of a more efficient use of the training data by using a fine-tuned object detector. The last row in table 1 shows the results attained by our complete model (with functional generalization). For the Full set, it achieves over 2.5\% absolute improvement over the best published work (Peyre et al.
Table 1: mAPs (\%) in the default setting for the HICO-Det dataset. Our model was trained with up to five neighbors. The last column is the total number of parameters in the proposed classification models.

\begin{tabular}{lccc|c}
\hline Method & $\begin{array}{c}\text { Full } \\
(600)\end{array}$ & $\begin{array}{c}\text { Rare } \\
(138)\end{array}$ & $\begin{array}{c}\text { Non-Rare } \\
(462)\end{array}$ & $\begin{array}{c}\text { Params. } \\
\text { (millions) }\end{array}$ \\
\hline (Shen et al. 2018) & 6.46 & 4.24 & 7.12 & - \\
(Chao et al. 2017) & 7.81 & 5.37 & 8.54 & - \\
(Gkioxari et al. 2017) & 9.94 & 7.16 & 10.77 & - \\
(Xu et al. 2018) & 9.97 & 7.11 & 10.83 & - \\
(Qi et al. 2018) & 13.11 & 9.34 & 14.23 & - \\
(Xu et al. 2019) & 14.70 & 13.26 & 15.13 & - \\
(Gao, Zou, and Huang 2018) & 14.84 & 10.45 & 16.15 & 48.1 \\
(Wang et al. 2019) & 16.24 & 11.16 & 17.75 & - \\
(Gupta, Schwing, and Hoiem 2019) & 17.18 & 12.17 & 18.68 & 9.2 \\
(Li et al. 2019) & 17.22 & 13.51 & 18.32 & 35.0 \\
(Zhou and Chi 2019) & 17.35 & 12.78 & 18.71 & - \\
(Wan et al. 2019) & 17.46 & 15.65 & 18.00 & - \\
(Peyre et al. 2019) & 19.40 & 15.40 & 20.75 & 21.8 \\
\hline Ours & $\mathbf{2 1 . 9 6}$ & $\mathbf{1 6 . 4 3}$ & $\mathbf{2 3 . 6 2}$ & $\mathbf{3 . 1}$ \\
\hline
\end{tabular}

2019). Our model also gives an mAP of $16.43 \%$ for Rare classes compared to the existing best of $15.65 \%$ (Wan et al. 2019). The performance, along with the simplicity, of our model is a remarkable strength and reveals that existing methods may be over-engineered.

Comparison of number of parameters. In table 1, we also compare the number of parameters in four recent models against our model. With far fewer parameters, our model achieves better performance. For example, compared to the current state-of-the-art model which contains 62.7 million parameters and achieves only $19.40 \%$ mAP, our model contains just 51.1 million parameters and reaches an mAP of $21.96 \%$. Ignoring the object detectors, our model introduces just 3.1 million new parameters. (Due to lack of specific details in previous papers, we have made some conservative assumptions which we list in the supplementary material.) In addition, the approaches in (Gupta, Schwing, and Hoiem 2019) and ( $\mathrm{Li}$ et al. 2019) require pose estimation models too. The numbers listed in table 1 do not count these parameters. The strength of our method is the simple and intuitive way of thinking about the problem.

Next, we show how a generic object detector can be used to detect novel interactions, even those involving objects not present in the training set. We will use an off-the-shelf Faster RCNN which is trained on OpenImages and is capable of detecting 545 object categories. This detector uses an Inception ResNet-v2 with atrous convolutions as its base network.

\section{Zero-shot HOI Detection}

(Shen et al. 2018) take the idea of zero-shot object detection further and try to detect previously unseen human-object relationships in images. The aim is to detect interactions for which no images are available during training. In this section, we show that our method offers significant improvements over (Shen et al. 2018) for zero-shot HOI detection.

Seen object scenario. We first consider the same setting as (Shen et al. 2018). We select 120 relationship triplets ensuring that every object involved in these 120 relationships 
Table 2: mAPs (\%) in the default setting for ZSD. This is the seen object setting, i.e., all the objects have been seen.

\begin{tabular}{lccc}
\hline Method & $\begin{array}{c}\text { Unseen } \\
(120 \text { classes })\end{array}$ & $\begin{array}{c}\text { Seen } \\
(480)\end{array}$ & $\begin{array}{c}\text { All } \\
(600)\end{array}$ \\
\hline (Shen et al. 2018) & 5.62 & - & 6.26 \\
\hline Ours & $\mathbf{1 1 . 3 1} \pm 1.03$ & $\mathbf{1 2 . 7 4} \pm 0.34$ & $\mathbf{1 2 . 4 5} \pm 0.16$ \\
\hline
\end{tabular}

Table 3: mAPs (\%) in the unseen object setting for ZSD. This is the unseen object setting where the trained model for interaction recognition has not seen any examples of some object classes.

\begin{tabular}{lccc}
\hline Method & $\begin{array}{c}\text { Unseen } \\
(100 \text { classes })\end{array}$ & $\begin{array}{c}\text { Seen } \\
(500)\end{array}$ & $\begin{array}{c}\text { All } \\
(600)\end{array}$ \\
\hline Ours & 11.22 & 14.36 & 13.84 \\
\hline
\end{tabular}

occurs in at least one of the remaining 480 triplets. We call this the "seen object" setting, i.e., the model sees all the objects involved but not all relationships. Later, we will introduce the "unseen object" where no relationships involving a set of objects will be observed during training.

Table 2 shows the performance of our approach in the "seen object" setting for 120 unseen triplets during training. Note that, since (Shen et al. 2018) have not release the list of classes publicly, we report the mean over 5 random sets of 120 unseen classes in table 2 . We achieve significant improvement over the prior method.

Unseen object scenario. We start by randomly selecting 12 objects from the 80 objects in HICO. We pick all relationships containing these objects. This gives us 100 relationship triplets which constitute the test (unseen) set. We train models using visual examples from only the remaining 500 categories. Table 3 gives results for our methods in this setting. We cannot compare with existing methods because none of them have the ability to detect HOIs in the unseen object scenario. We hope that our method will serve as a baseline for future research on this important problem.

In figure 4, we show that our model can detect interaction triplets with unseen objects. This is because we use a generic detector which can detect many more objects. We note, here, that there are some classes among the $80 \mathrm{COCO}$ classes which do not occur in OI. We willingly take the penalty for missing interactions with these objects in order to present a more robust system which not only works for the dataset of interest but is able to generalize to completely unseen interaction classes. We reiterate that none of the previous methods has the ability to detect HOIs in this scenario.

\section{Ablation Analysis}

The generic object detector used for zero-shot HOI detection can also be used in the supervised setting. For example, using this detector, we obtain an mAP of $14.35 \%$ on the Full set of HICO-Det. This is a competitive performance and is worse (table 1) than only the most recent works. This shows the strength of generalization. In this section, we provide
Table 4: HICO-Det performance (mAP \%) of the model with different number of neighbors considered for generalization.

\begin{tabular}{cccc}
\hline $\begin{array}{c}\mathbf{r} \\
\text { (Number of objects) }\end{array}$ & $\begin{array}{c}\text { Full } \\
(600 \text { classes })\end{array}$ & $\begin{array}{c}\text { Rare } \\
(138)\end{array}$ & $\begin{array}{c}\text { Non-Rare } \\
(462)\end{array}$ \\
\hline 0 & 12.72 & 7.57 & 14.26 \\
3 & 13.70 & 7.98 & 15.41 \\
5 & $\mathbf{1 4 . 3 5}$ & $\mathbf{9 . 8 4}$ & $\mathbf{1 5 . 6 9}$ \\
7 & 13.51 & 7.07 & 15.44 \\
\hline
\end{tabular}

Table 5: mAPs (\%) for different clustering methods.

\begin{tabular}{lccc}
\hline $\begin{array}{l}\text { Clustering } \\
\text { Algorithm }\end{array}$ & $\begin{array}{c}\text { Full } \\
\text { (600 classes) }\end{array}$ & $\begin{array}{c}\text { Rare } \\
(138)\end{array}$ & $\begin{array}{c}\text { Non-Rare } \\
(462)\end{array}$ \\
\hline K means & $\mathbf{1 4 . 3 5}$ & $\mathbf{9 . 8 4}$ & 15.69 \\
Agglomerative & 14.05 & 7.59 & $\mathbf{1 5 . 9 8}$ \\
Affinity Propagation & 13.49 & 7.53 & 15.28 \\
\hline
\end{tabular}

further analysis of our model with the generic detector.

Number of neighbors. Table 4 shows the effect of varying the number of neighboring objects which are added to the dataset for each training instance. The baseline (first row) is when no additional objects are added. This is when we rely only on the interactions present in the original dataset. We successively add interactions with neighboring objects to the training data and observe that the performance improves significantly. However, since the clusters are not perfect, adding more neighbors can start becoming harmful. Also, the training times increase rapidly. Therefore, we add five neighbors for each HOI instance in all our experiments.

Clustering method. To check if another clustering algorithm might be better, we create clusters using different algorithms. From table 5 we observe that K-means clustering leads to the best performance. Hierarchical agglomerative clustering also gives close albeit lower performance.

Importance of features. Further ablation studies (table 6) show that removing $f_{g}, f_{h}$, or semantic word-vectors $w_{h}, w_{o}$ from the functional generalization module leads to a reduction in performance. For example, training the model without the geometric feature $f_{g}$ gives an mAP of $12.43 \%$ and training the model without $f_{h}$ in the generalization module gives an mAP of just $12.15 \%$. In particular, the performance for Rare classes is quite low. This shows that these features are important for detecting Rare HOIs. Note that, removing $w_{o}$ means that there is no functional generalization.

\section{Dealing with Dataset Bias}

Dataset bias leads to models being biased towards particular classes (Torralba, Efros, and others 2011). In fact, bias in the training dataset is usually amplified by the models (Zhao et al. 2017; Anne Hendricks et al. 2018). Our proposed method can be used as a way to overcome the dataset bias problem. To illustrate this, we use metrics proposed in (Zhao et al. 2017) to quantitatively study model bias.

We consider a set of (object, predicate) pairs $\mathcal{Q}=\left\{\left(o_{1}, p_{1}\right), \ldots,\left(o_{2}, p_{2}\right)\right\}$. For each pair in $\mathcal{Q}$, we con- 


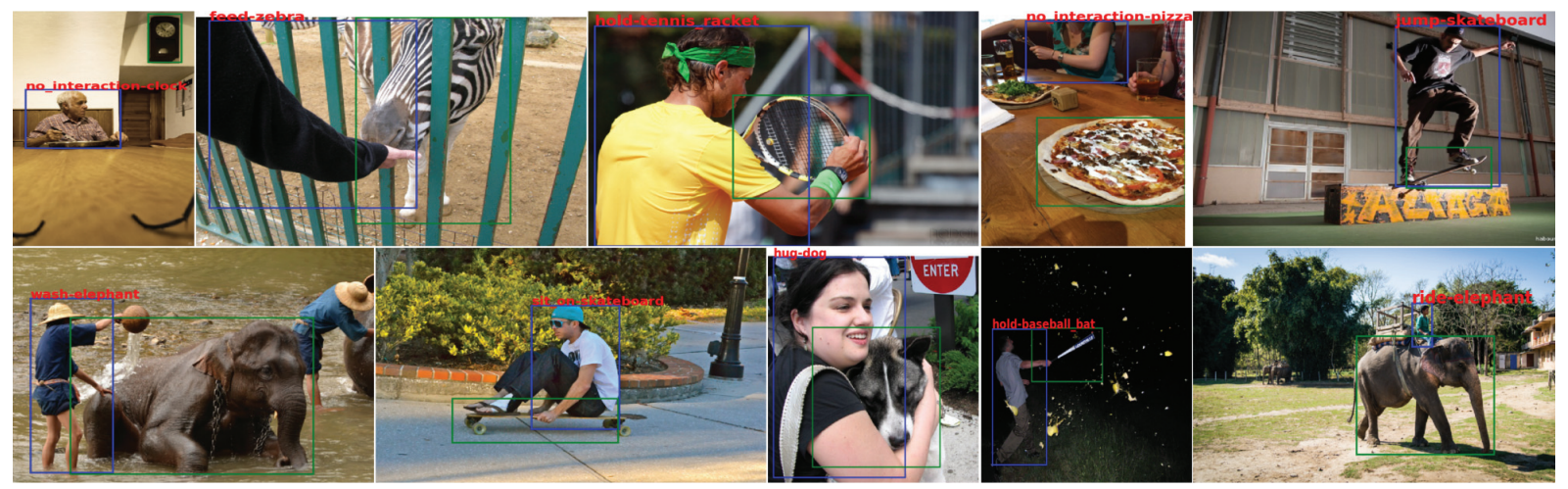

Figure 4: Some HOI detections in the unseen object ZSD setting. Our model has not seen any image with the objects shown above during training. (We show some mistakes made by the model in the supplementary material.)

Table 6: Ablation studies (mAP \%).

\begin{tabular}{lccc}
\hline Setting & $\begin{array}{c}\text { Full } \\
\text { (600 classes) }\end{array}$ & $\begin{array}{c}\text { Rare } \\
(138)\end{array}$ & $\begin{array}{c}\text { Non-Rare } \\
(462)\end{array}$ \\
\hline Base & $\mathbf{1 4 . 3 5}$ & $\mathbf{9 . 8 4}$ & $\mathbf{1 5 . 6 9}$ \\
Base $-f_{h}$ & 12.15 & 4.87 & 14.33 \\
Base $-f_{g}$ & 12.43 & 8.02 & 13.75 \\
Base $-w_{h}-w_{o}$ & 12.23 & 5.23 & 14.32 \\
\hline
\end{tabular}

sider two scenarios: (1) the training set is heavily biased against the pair; (2) the training set is heavily biased towards the pair. For generating the training sets for a pair $q_{i}=\left\{o_{i}, p_{i}\right\} \in \mathcal{Q}$, for the first scenario, we remove all training samples containing the pair $q_{i}$ and keep all other samples for the object. Similarly, for the second scenario, we remove all training samples containing $o_{i}$ except those containing the pair $q_{i}$. For the pair, $q_{i}$ the test set bias is $b_{i}$ (We adopt the definition of bias from (Zhao et al. 2017). See supplementary material for more details.). Given two models, the one with bias closer to test set bias is considered better. We show that our approach of augmenting the dataset brings the model bias closer to the test set bias. In particular, we consider $\mathcal{Q}=\{$ (horse, ride), (cup, hold) $\}$, such that $b_{1}=0.275$ and $b_{2}=0.305$.

In the first scenario, baseline models trained on biased datasets have biases 0.124 and 0.184 for (horse, ride) and (cup, hold) respectively. Note that these are less than the test set biases because of the heavy bias against these pairs in their respective training sets. Next, we train models by augmenting the training sets using our methodology for only one neighbor of each object. Models trained on these new sets have biases 0.130 and 0.195 . That is, our approach leads to a reduction in the bias against these pairs.

Similarly, for the second scenario, baseline models trained on the biased datasets have biases 0.498 and 0.513 for (horse, ride) and (cup, hold) respectively. Training models on datasets de-biased by our approach give biases 0.474 and 0.50 . In this case, our approach leads to a reduction in the bias towards these pairs.

\section{Discussion and Conclusion}

We discuss some limitations of the proposed approach. First, we assume that all predicates follow functional similarities. However, some predicates might only apply to particular objects. For example, you can blow a cake, but not a donut which is functionally similar to cake. Our current model does not capture such constraints. Further work can focus on trying to explicitly incorporate such priors into the model. A related limitation is the independence assumption on predicates. In fact, some predicates are completely dependent. For example, straddle usually implies sit_on for bicycles or horses. However, due to the in-exhaustive labeling of the datasets, we (and most previous work) ignore this dependence. Approaches exploiting co-occurrences of predicates can help overcome this problem.

Conclusion. We have presented a way to enhance HOI detection by incorporating the common-sense idea that human-object interactions look similar for functionally similar objects. Our method is able to detect previously unseen (zero-shot) human-object relationships. We have provided experimental validation for our claims and have reported state-of-the-art results for the problem. However, there are still several issues that need to be solved to advance the understanding of the problem and improve performance of models.

\section{Acknowledgement}

This project was supported by the Intelligence Advanced Research Projects Activity (IARPA) via Department of Interior/Interior Business Center (DOI/IBC) contract number D17PC00345 and by DARPA via ARO contract number W911NF2020009. The U.S. Government is authorized to reproduce and distribute reprints for Governmental purposes not withstanding any copyright annotation thereon.

Disclaimer: The views and conclusions contained herein are those of the authors and should not be interpreted as necessarily representing the official policies or endorsements, either expressed or implied of IARPA, DOI/IBC or the U.S. Government. 


\section{Supplementary Material}

\section{Representative clusters}

We claim that the objects in the same cluster can be considered functionally similar. Representative clusters are: ['Mug', 'Pitcher', 'Teapot', 'Kettle', 'Jug'], and ['Elephant', 'Dinosaur', 'Cattle', 'Horse', 'Giraffe', 'Zebra', 'Rhinoceros', 'Mule', 'Camel', 'Bull']. Clearly, our clusters contain functionally similar objects. During training, for augmentation we replace the object in a training sample by other objects from the same cluster. For example, given a training sample for ride-elephant, we generate new samples by replacing elephant by horse or camel.

\section{Performance with COCO Detector}

With the original COCO-trained detector, our method gives an mAP of $16.96,11.73$, and $18.52 \%$ respectively for Full, Rare and Non-Rare sets (up from 14.37, 7.83, 16.33\% without functional generalization). This performance improvement in even more significant due to the use of an order of magnitude fewer parameters than existing approaches. In addition, the proposed approach could be incorporated with any existing method as shown in the next section.

\section{Bonus Experiment: Visual Model}

Our generalization module can be complementary to existing approaches. To illustrate this, we consider a simple visual module shown in figure $\mathrm{S} 1$. It takes the union of $b_{h}$ and $b_{o}$ and crops the union box from the image. It passes the cropped union box through a CNN (ResNet-50). The feature obtained, $f_{u}$ is concatenated with $f_{h}$ and $f_{o}$ and passed through two FC layers. This module and the generalization module independently predict the probabilities for predicates and the final prediction is the average of the two. Using the generic object detector, the combined model gives an mAP of $15.82 \%$ on the Full HICO-Det dataset (the visual model separately gives $14.11 \%$ ). This experiment shows that functional generalization proposed in this paper is complementary to existing works which rely on purely visual data. Using our generalization module in conjugation with other existing methods can lead to performance improvements.

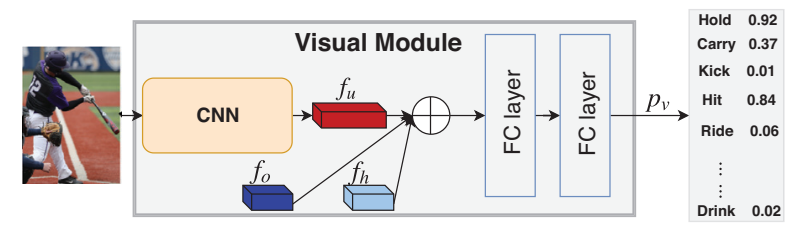

Figure S1: Simple visual module.

\section{Assumptions about number of parameters}

Some works (Gupta, Schwing, and Hoiem 2019) have all the details necessary for the computation in their manuscript, while some (Gao, Zou, and Huang 2018; Li et al. 2019; Peyre et al. 2019) fail to mention the specifics. Hence, we made the following assumptions while estimating the number of parameters. Note that only those methods, where sufficient details weren't mentioned in the paper, are discussed. Since all of the methods use an object detector in the first step, we compute the number of parameters introduced by the detector. Table $\mathrm{S} 1$ shows the number of parameters estimated for each method.

Table S1: Estimated parameters (in millions) for the detectors used in a few of the state-of-the-art methods. ("R-" stands for "ResNet")

\begin{tabular}{c|c|c}
\hline Method & Detector & Params \\
\hline (Gao, Zou, and Huang 2018) & FPN R-50 & 40.9 \\
(Gupta, Schwing, and Hoiem 2019) & Faster-RCNN R-152 & 63.7 \\
(Li et al. 2019) & Faster-RCNN R-50 & 29 \\
(Peyre et al. 2019) & FPN R-50 & 40.9 \\
Ours & Faster-RCNN R-101 & 48 \\
\hline
\end{tabular}

ICAN. Authors in (Gao, Zou, and Huang 2018) use two fully connected layers in each of the human, object, and pairwise streams, but the details of the hidden layers were not mentioned in their work. The feature dimensions of the human and object stream are 3072, while for the pairwise stream it is 5408. To make a conservative estimate, we assume the dimensions of the hidden layers to be 1024 and 512 for the human and object stream. For the pairwise stream we assume dimensions of 2048 and 512 for the hidden layers. We end up with an estimated total of $48.1 \mathrm{M}$ parameters for their architecture. This gives the total parameters for their method to be 89M (48.1+ 40.9 (Detector; see table S1)).

Interactiveness Prior. Li et al. (Li et al. 2019) used a FasterRCNN (Ren et al. 2015) based detector with a ResNet50 backbone architecture. In their proposed approach, they have 10 MLPs (multi-layer perceptrons) with two layers each and 3 fully connected (FC) layers. Out of the 10 MLPs, we estimated 6 of them to have an input dimension of 2048 , 3 of them to have 1024 and one of them 3072. The dimension of hidden layers was given to be 1024 for all the 10 MLPs. The 3 FC layers have input dimensions of 1024 and an output dimension 117 . This gives the number of parameters utilized as $35 \mathrm{M}$. Their total number of parameters = 64M $(35+29$ (detector)).

Peyre et al. Peyre et al. used a FPN (Lin et al. 2017) detector with a ResNet-50 backbone. They have a total of 9 MLPs with two hidden layers each, and 3 FC layers. The input dimension of the FC layers is 2048 and the output dimension is 300.6 of the 9 MLPs have an input dimension of 300 and an output dimension of 1024 . Another 2 of the 9 MLPs have input dimension of 1000 and 900 respectively. Their output dimension is 1024 . We assume the dimensions of the hidden layers in all these MLPs to be 1024 and 1024. The last of the 9 MLPs has an input dimension of 8 and an output dimension of 400 . We assume a hidden layer of dimension 256 for this MLP. This brings the estimated parameter used to $21.8 \mathrm{M}$ and their total parameter count $=\mathbf{6 2 . 7 M}(21.8+$ 40.9 (detector)). 

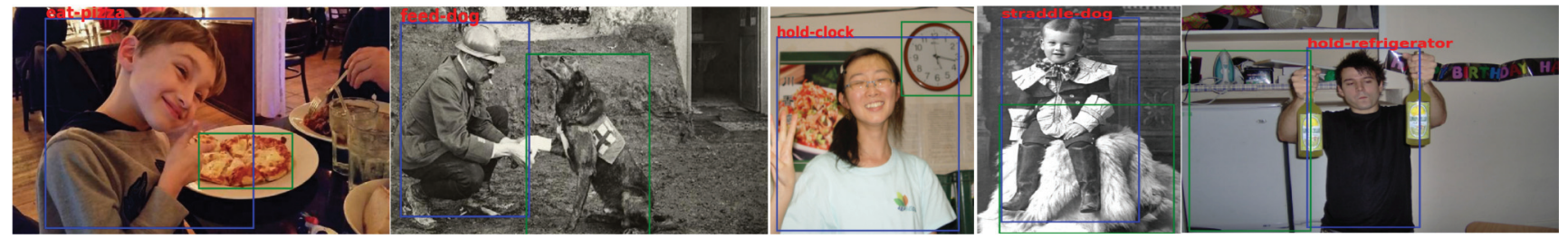

Figure S2: Some incorrect HOI detections in the unseen object ZSD setting. Our model has not seen any image with the objects shown above during training.

\section{Failure cases}

Figure S2 shows some incorrect detections made by our model in the unseen object zero-shot scenario. Most of these incorrect detections are very close to being correct. For example, in the first image, it's very difficult, even for humans to figure out that the person is not eating the pizza on the plate. In the third and last images, the persons are holding something, just not the object under consideration. Our current model, cannot ignore other objects present in the scene which lie very close to the person or the object of interest. This is an area for further research.

\section{Bias details}

Adopting the bias metric from (Zhao et al. 2017), we define the bias for a verb-object pair, $\left(v_{*}, o\right)$ in a set as:

$$
b_{s}\left(v_{*}, o\right)=\frac{c_{s}\left(v_{*}, o\right)}{\sum_{v} c_{s}(v, o)}
$$

where, $c_{s}(v, o)$ is the number of instances of the pair $(v, o)$ in the set, $s$. This measure can be used to quantify the bias for a verb-object pair in a dataset or for a model's prediction. For a dataset, $\mathcal{D}, c_{\mathcal{D}}(v, o)$ gives the number of instances of $(v, o)$ pairs in it. Therefore, $b_{\mathcal{D}}$ represents the bias for the pair $\left(v_{*}, o\right)$ in the dataset. A low value $(\approx 0)$ of $b_{\mathcal{D}}$ means that the set is heavily biased against the pair while a high value $(\approx 1)$ means that it is heavily biased towards the pair.

Similarly, we can define the bias of a model by considering the model's predictions as the dataset under consideration. For example, suppose that the model under consideration gives the predictions $\mathcal{P}$ for the dataset $\mathcal{D}$. We can define the model's bias as:

$$
b_{\mathcal{P}}\left(v_{*}, o\right)=\frac{c_{\mathcal{P}}\left(v_{*}, o\right)}{\sum_{v} c_{\mathcal{P}}(v, o)}
$$

where, $c_{\mathcal{P}}(v, o)$ gives the number of instances of the pair $(v, o)$ in the set of the model's predictions $\mathcal{P}$.

A perfect model is one whose bias, $b_{\mathcal{P}}\left(v_{*}, o\right)$ is equal to the dataset bias $b_{\mathcal{D}}\left(v_{*}, o\right)$. However, due to bias amplification (Zhao et al. 2017; Anne Hendricks et al. 2018), most models will have a higher/lower bias than the test dataset depending on the training set bias. That is, if the training set is heavily biased towards (resp. against) a pair, then the model's predictions will be more heavily biased towards (resp. against) that pair for the test set. The aim of a bias reduction method should be to bring the model's bias closer to the test set bias. Our experiments in the paper showed that our proposed algorithm is able to reduce the gap between the test set bias and the model prediction bias.

\section{References}

Anne Hendricks, L.; Burns, K.; Saenko, K.; Darrell, T.; and Rohrbach, A. 2018. Women also snowboard: Overcoming bias in captioning models. In Proceedings of the European Conference on Computer Vision (ECCV), 771-787.

Bansal, A.; Sikka, K.; Sharma, G.; Chellappa, R.; and Divakaran, A. 2018. Zero-shot object detection. In The European Conference on Computer Vision (ECCV).

Chao, Y.-W.; Wang, Z.; He, Y.; Wang, J.; and Deng, J. 2015. Hico: A benchmark for recognizing human-object interactions in images. In Proceedings of the IEEE International Conference on Computer Vision, 1017-1025.

Chao, Y.-W.; Liu, Y.; Liu, X.; Zeng, H.; and Deng, J. 2017. Learning to detect human-object interactions. arXiv preprint arXiv:1702.05448.

Delaitre, V.; Sivic, J.; and Laptev, I. 2011. Learning personobject interactions for action recognition in still images. In Advances in neural information processing systems, 15031511.

Desai, C., and Ramanan, D. 2012. Detecting actions, poses, and objects with relational phraselets. In European Conference on Computer Vision, 158-172. Springer.

Fang, H.-S.; Cao, J.; Tai, Y.-W.; and Lu, C. 2018. Pairwise body-part attention for recognizing human-object interactions. arXiv preprint arXiv:1807.10889.

Gao, C.; Zou, Y.; and Huang, J.-B. 2018. ican: Instancecentric attention network for human-object interaction detection. arXiv preprint arXiv:1808.10437.

Gibson, J. 1979. The theory of affordances the ecological approach to visual perception (pp. 127-143).

Gkioxari, G.; Girshick, R.; Dollár, P.; and He, K. 2017. Detecting and recognizing human-object interactions. arXiv preprint arXiv:1704.07333.

Gupta, A., and Davis, L. S. 2007. Objects in action: An approach for combining action understanding and object perception. In Computer Vision and Pattern Recognition, 2007. CVPR'07. IEEE Conference on, 1-8. IEEE.

Gupta, S., and Malik, J. 2015. Visual semantic role labeling. arXiv preprint arXiv:1505.04474.

Gupta, A.; Kembhavi, A.; and Davis, L. S. 2009. Observing human-object interactions: Using spatial and functional compatibility for recognition. IEEE Transactions on Pattern Analysis and Machine Intelligence 31(10):1775-1789. 
Gupta, T.; Schwing, A.; and Hoiem, D. 2019. No-frills human-object interaction detection: Factorization, layout encodings, and training techniques. The IEEE International Conference on Computer Vision (ICCV) 9677-9685.

Huang, J.; Rathod, V.; Sun, C.; Zhu, M.; Korattikara, A.; Fathi, A.; Fischer, I.; Wojna, Z.; Song, Y.; Guadarrama, S.; et al. 2017. Speed/accuracy trade-offs for modern convolutional object detectors. In IEEE CVPR, volume 4.

Kato, K.; Li, Y.; and Gupta, A. 2018. Compositional learning for human object interaction. In Proceedings of the European Conference on Computer Vision (ECCV), 234-251.

Kodirov, E.; Xiang, T.; and Gong, S. 2017. Semantic autoencoder for zero-shot learning. arXiv preprint arXiv:1704.08345.

Kolesnikov, A.; Lampert, C. H.; and Ferrari, V. 2018. Detecting visual relationships using box attention. arXiv preprint arXiv:1807.02136.

Krishna, R.; Zhu, Y.; Groth, O.; Johnson, J.; Hata, K.; Kravitz, J.; Chen, S.; Kalantidis, Y.; Li, L.-J.; Shamma, D. A.; et al. 2017. Visual genome: Connecting language and vision using crowdsourced dense image annotations. International Journal of Computer Vision 123(1):32-73.

Kuznetsova, A.; Rom, H.; Alldrin, N.; Uijlings, J.; Krasin, I.; Pont-Tuset, J.; Kamali, S.; Popov, S.; Malloci, M.; Duerig, T.; and Ferrari, V. 2018. The open images dataset v4: Unified image classification, object detection, and visual relationship detection at scale. arXiv:1811.00982.

Li, Y.-L.; Zhou, S.; Huang, X.; Xu, L.; Ma, Z.; Fang, H.S.; Wang, Y.; and Lu, C. 2019. Transferable interactiveness knowledge for human-object interaction detection. The IEEE Conference on Computer Vision and Pattern Recognition $(C V P R)$.

Lin, T.-Y.; Maire, M.; Belongie, S.; Hays, J.; Perona, P.; Ramanan, D.; Dollár, P.; and Zitnick, C. L. 2014. Microsoft coco: Common objects in context. In European conference on computer vision, 740-755. Springer.

Lin, T.; Dollar, P.; Girshick, R.; He, K.; Hariharan, B.; and Belongie, S. 2017. Feature pyramid networks for object detection. In 2017 IEEE Conference on Computer Vision and Pattern Recognition (CVPR), 936-944. Los Alamitos, CA, USA: IEEE Computer Society.

Lu, C.; Krishna, R.; Bernstein, M.; and Fei-Fei, L. 2016. Visual relationship detection with language priors. In European Conference on Computer Vision, 852-869. Springer.

Mikolov, T.; Sutskever, I.; Chen, K.; Corrado, G. S.; and Dean, J. 2013. Distributed representations of words and phrases and their compositionality. In NIPS.

Miller, G. A. 1995. Wordnet: a lexical database for english. Communications of the ACM 38(11):39-41.

Peyre, J.; Laptev, I.; Schmid, C.; and Sivic, J. 2019. Detecting unseen visual relations using analogies. The IEEE International Conference on Computer Vision (ICCV).

Qi, S.; Wang, W.; Jia, B.; Shen, J.; and Zhu, S.-C. 2018. Learning human-object interactions by graph parsing neural networks. arXiv preprint arXiv:1808.07962.
Rahman, S.; Khan, S.; and Porikli, F. 2018. Zero-shot object detection: Learning to simultaneously recognize and localize novel concepts. arXiv preprint arXiv:1803.06049.

Ren, S.; He, K.; Girshick, R.; and Sun, J. 2015. Faster rcnn: Towards real-time object detection with region proposal networks. In NIPS, 91-99.

Sadeghi, M. A., and Farhadi, A. 2011. Recognition using visual phrases. In Computer Vision and Pattern Recognition (CVPR), 2011 IEEE Conference on, 1745-1752. IEEE.

Shen, L.; Yeung, S.; Hoffman, J.; Mori, G.; and Fei-Fei, L. 2018. Scaling human-object interaction recognition through zero-shot learning. In 2018 IEEE Winter Conference on Applications of Computer Vision (WACV), 1568-1576. IEEE.

Torralba, A.; Efros, A. A.; et al. 2011. Unbiased look at dataset bias. In $C V P R$, volume 1, 7. Citeseer.

Wan, B.; Zhou, D.; Liu, Y.; Li, R.; and He, X. 2019. Poseaware multi-level feature network for human object interaction detection. In The IEEE International Conference on Computer Vision (ICCV).

Wang, T.; Anwer, R. M.; Khan, M. H.; Khan, F. S.; Pang, Y.; Shao, L.; and Laaksonen, J. 2019. Deep contextual attention for human-object interaction detection. In The IEEE International Conference on Computer Vision (ICCV).

Xian, Y.; Schiele, B.; and Akata, Z. 2017. Zero-shot learning-the good, the bad and the ugly. arXiv preprint arXiv:1703.04394.

Xu, B.; Li, J.; Wong, Y.; Kankanhalli, M. S.; and Zhao, Q. 2018. Interact as you intend: Intention-driven human-object interaction detection. arXiv preprint arXiv:1808.09796.

Xu, B.; Wong, Y.; Li, J.; Zhao, Q.; and Kankanhalli, M. S. 2019. Learning to detect human-object interactions with knowledge. In The IEEE Conference on Computer Vision and Pattern Recognition (CVPR).

Yao, B., and Fei-Fei, L. 2010. Grouplet: A structured image representation for recognizing human and object interactions. In Computer Vision and Pattern Recognition (CVPR), 2010 IEEE Conference on, 9-16. IEEE.

Yao, B.; Jiang, X.; Khosla, A.; Lin, A. L.; Guibas, L.; and Fei-Fei, L. 2011. Human action recognition by learning bases of action attributes and parts. In Computer Vision (ICCV), 2011 IEEE International Conference on, 13311338. IEEE.

Zhao, J.; Wang, T.; Yatskar, M.; Ordonez, V.; and Chang, K.-W. 2017. Men also like shopping: Reducing gender bias amplification using corpus-level constraints. arXiv preprint arXiv:1707.09457.

Zhou, P., and Chi, M. 2019. Relation parsing neural network for human-object interaction detection. In The IEEE International Conference on Computer Vision (ICCV).

Zhuang, B.; Liu, L.; Shen, C.; and Reid, I. 2017a. Towards context-aware interaction recognition. arXiv preprint arXiv:1703.06246.

Zhuang, B.; Wu, Q.; Shen, C.; Reid, I.; and Hengel, A. v. d. 2017b. Care about you: towards large-scale human-centric visual relationship detection. arXiv preprint arXiv:1705.09892. 